\title{
Sustained effectiveness of a multifaceted intervention to reduce potentially inappropriate prescribing in older patients in primary care (OPTI-SCRIPT study)
}

Barbara Clyne ${ }^{1 *}$, Susan M. Smith ${ }^{1}$, Carmel M. Hughes ${ }^{2}$, Fiona Boland ${ }^{1}$, Janine A. Cooper ${ }^{1,2}$, Tom Fahey ${ }^{1}$ and on behalf of the OPTI-SCRIPT study team

\begin{abstract}
Background: Potentially inappropriate prescribing (PIP) is common in older people in primary care and can result in increased morbidity, adverse drug events and hospitalisations. We previously demonstrated the success of a multifaceted intervention in decreasing PIP in primary care in a cluster randomised controlled trial (RCT).

Objective: We sought to determine whether the improvement in PIP in the short term was sustained at 1-year follow-up.

Methods: A cluster RCT was conducted with 21 GP practices and 196 patients (aged $\geq 70$ ) with PIP in Irish primary care. Intervention participants received a complex multifaceted intervention incorporating academic detailing, medicine review with web-based pharmaceutical treatment algorithms that provide recommended alternative treatment options, and tailored patient information leaflets. Control practices delivered usual care and received simple, patient-level PIP feedback. Primary outcomes were the proportion of patients with PIP and the mean number of potentially inappropriate prescriptions at 1-year follow-up. Intention-to-treat analysis using random effects regression was used.
\end{abstract}

Results: All 21 GP practices and 186 (95\%) patients were followed up. We found that at 1-year follow-up, the significant reduction in the odds of PIP exposure achieved during the intervention was sustained after its discontinuation (adjusted OR 0.28, $95 \% \mathrm{Cl} 0.11$ to $0.76, P=0.01$ ). Intervention participants had significantly lower odds of having a potentially inappropriate proton pump inhibitor compared to controls (adjusted OR 0.40, $95 \% \mathrm{Cl}$ 0.17 to $0.94, P=0.04)$.

Conclusion: The significant reduction in the odds of PIP achieved during the intervention was sustained after its discontinuation. These results indicate that improvements in prescribing quality can be maintained over time.

Trial registration: Current controlled trials ISRCTN41694007.

Keywords: Randomised controlled trial, Potentially inappropriate prescribing, Primary health care

\footnotetext{
* Correspondence: barbaraclyne@rcsi.ie

${ }^{1}$ HRB Centre for Primary Care Research, Department of General Practice,

Royal College of Surgeons in Ireland (RCSI), 123 St. Stephens Green, Dublin 2,

Republic of Ireland

Full list of author information is available at the end of the article
} 


\section{Introduction}

Medication use in older people can improve wellbeing and quality of life; however, drug-related problems such as medication errors and adverse drug events (ADEs) are common [1]. Evidence suggests that prescribing in this population can be potentially inappropriate [2]. Medications are termed potentially inappropriate where their risks outweigh the benefits and when a safer therapeutic alternative is available [3]. Potentially inappropriate prescribing (PIP) is estimated to affect between 10 and $50 \%$ of community dwelling older people internationally, increasing the risk of morbidity, ADEs, hospitalisations and health expenditure in this population [2, 4-8].

Interventions such as computerised decision support systems (CDSS), pharmacist interventions and multifaceted interventions may be useful strategies in reducing PIP in different health care settings [9-12]. We have previously demonstrated that a multifaceted intervention was effective in decreasing PIP in older patients in primary care using a short-term followup, on intervention completion at 4-6 months [13]. The short-term results indicated that patients in the intervention group had significantly lower odds of having PIP than patients in the control group (adjusted odds ratio (OR) 0.32, $95 \%$ confidence interval (CI) 0.15 to $0.70, P=0.02$ ). The mean number of PIP drugs in intervention was 0.70 , compared to 1.18 in control $(P=0.02)$. The intervention was effective in reducing proton pump inhibitor prescribing (adjusted OR $0.30,95 \%$ CI 0.14 to $0.68, P=0.04$ ), but not other drug classes [13].

The use of such short-term follow-up is a common criticism, raising concerns about the long-term sustainability of such interventions [2]. Even when inappropriate medications are ceased, evidence indicates that they might be restarted, particularly where multiple prescribers are involved [14]. Posttrial follow-up is therefore recommended to assess if short-term changes persist. Post-trial follow-up is necessary to assess if trial effects diminish, remain constant or increase after the randomised interventions are formally discontinued. The objective of this study was to determine whether the immediate improvement in PIP in the short-term was sustained at 1 year follow-up.

\section{Methods}

A cluster randomised controlled trial (RCT) was conducted in Irish primary care to alter general practitioner (GP) PIP-related prescribing. The study protocol, intervention development and short-term outcomes (intervention completion at 4-6 months) have been reported in detail previously and are summarised in brief below $[13,15,16]$. The Research Ethics Committee of the Irish College of General Practitioners (ICGP) approved the study.

\section{Recruitment and randomisation}

A total of 65 general practices from the greater Dublin area were invited to participate in this study with 21 (32\%) consenting. Consenting practices were assisted by the study team in identifying and recruiting approximately 10 patients per practice. Patients were eligible where they were aged $\geq 70$ years and had pre-existing PIP (as determined by having one or more pre-specified PIP indicators, see Appendix [16]). In total, 196 patients were recruited. Fifty-three per cent of the pre-specified indicators were present in this population. Practices were allocated using minimisation to intervention or control after baseline data collection. It was not possible to blind patients or GPs to allocations; however, the outcome assessor was blinded.

\section{Intervention and control groups}

The intervention group (11 practices, 99 patients) received a multifaceted intervention involving academic detailing with a pharmacist on how to conduct GPled medicines review with participating patients. Medicine reviews were supported by web-based pharmaceutical treatment algorithms for GPs providing evidence-based alternative treatment options to PIP drugs and tailored patient information leaflets [15]. The control group (10 practices, 97 patients $)$ delivered usual care and received one-off simple patientlevel PIP feedback (see Table 1).

Formal support for the intervention finished at 6 months (intervention completion), and all practices (intervention and control) received a report summarising participating patients and their PIP profile for use for internal audit purposes. GPs and patients returned to their usual practice, with no attempt to encourage further medicine review or alteration to medications.

\section{Outcomes and statistical analysis}

Outcome data were collected at 1-year postintervention completion (i.e. 1 year after formal support for the intervention stopped). Patient records were used to collect outcome data, i.e. medication and health service use data for all eligible participants. Data was extracted by review of the patient's chart (either electronic or paper based depending on the practice system). 
Table 1 Summary of OPTI-SCRIPT intervention and control groups

\begin{tabular}{|c|c|}
\hline Intervention & The intervention consisted of: \\
\hline & $\begin{array}{l}\text { (1) Academic detailing with a } \\
\text { pharmacist } \\
\text { One session ( } 30 \text { min) where } \\
\text { a pharmacist visited the practice } \\
\text { to discuss PIP, medicine review and } \\
\text { the web-based pharmaceutical } \\
\text { treatment algorithms } \\
\text { (2) Medicine review with } \\
\text { web-based } \\
\text { pharmaceutical treatment } \\
\text { algorithms. } \\
\text { GPs were asked to conduct } \\
\text { one eview per patient using } \\
\text { the web-based platform to } \\
\text { guide them through the } \\
\text { process. The GP was } \\
\text { presented with the specific } \\
\text { PIP drug(s) for each patient, } \\
\text { and for each PIP drug, there } \\
\text { was a treatment algorithm } \\
\text { with the following structure: } \\
\text { a. The individual PIP with reason } \\
\text { for concern } \\
\text { b. Alternative pharmacological } \\
\text { and non-pharmacological } \\
\text { treatment options } \\
\text { c. Background information } \\
\text { (where relevant) }\end{array}$ \\
\hline & $\begin{array}{l}\text { (3) Patient information leaflets to } \\
\text { give to patients during the review. } \\
\text { Each leaflet: } \\
\text { a. Described the PIP and the } \\
\text { reasons as to why it may be } \\
\text { inappropriate } \\
\text { b. Outlined the alternative } \\
\text { pharmacological and } \\
\text { non-pharmacological } \\
\text { therapies GPs may offer }\end{array}$ \\
\hline Control & $\begin{array}{l}\text { Control practices delivered } \\
\text { usual care. Usual care for } \\
\text { public general medical } \\
\text { services (GMS) patients allows } \\
\text { GPs to give a prescription on } \\
\text { a monthly or three monthly } \\
\text { basis. } \\
\text { Control practices received } \\
\text { simple patient-level PIP postal } \\
\text { feedback in the form of a list } \\
\text { summarising the medication } \\
\text { class to which the individual } \\
\text { patient's potentially inappropriate } \\
\text { medication belonged. } \\
\text { Control practices did not receive } \\
\text { an academic detailing } \\
\text { visit or were not prompted to } \\
\text { carry out medicines review } \\
\text { with the individual patients. }\end{array}$ \\
\hline
\end{tabular}

GMS general medical services, PIP potentially inappropriate prescribing Source: Clyne et al. [13]
The primary outcomes were the proportion of patients with PIP and the mean number of PIP drugs. The proportion of patients with PIP is presented and was analysed using a random effects logistic regression with the individual as the unit of analysis and the practice included as the random effect to control for the effects of clustering. Baseline covariates (age, gender, baseline number of PIP drugs, baseline number of repeat medications) and minimisation factors (number of GPs, practice location) were included in the model. The mean number of PIP drugs was calculated per group, and a mean difference calculated using a cluster level $t$ test. Intention-to-treat analysis using random effects regression was used.

Secondary outcomes assessed differences between intervention and control in relation to individual drugs (using random effects logistic regressions) and health service utilisation including the number of GP visits and in-patient days (using random effects multiple regressions).

\section{Results}

Figure 1 displays the flow of participants through the RCT. All GP practices and 186 (95\%) patients were followed up at 1 year. At baseline, receipt of proton pump inhibitors at maximum therapeutic dosage for more than 8 weeks was the most frequently occurring PIP, with $60 \%$ of participants having this indicator [13].

\section{Primary outcomes}

At 1-year follow-up, the proportion of patients with PIP drugs was 0.51 in the intervention group compared to 0.76 in the control group. Intervention group participants had significantly lower odds of having PIP than control participants (adjusted OR 0.28, $95 \%$ CI 0.11 to $0.76, P=0.01$ ) (Table 2). The mean number of PIP drugs in the intervention group was 0.61 (SD 0.7) compared to 1.03 (SD 0.8) in the control group $(P=0.01)$. Intervention participants had significantly lower odds of having a potentially inappropriate proton pump inhibitor compared to controls (adjusted OR 0.40, $95 \%$ CI 0.17 to $0.94, s=0.04$ ). No statistically significant differences were found for other drug-specific outcomes.

\section{New PIP}

Between baseline and 1-year follow-up, a total of 34 new instances of PIP were identified in 30 patients (13\% of total sample). In the intervention group, 12 (13\%) participants had a total of 16 new instances of PIP, compared to 18 (20\%) participants with 18 new 


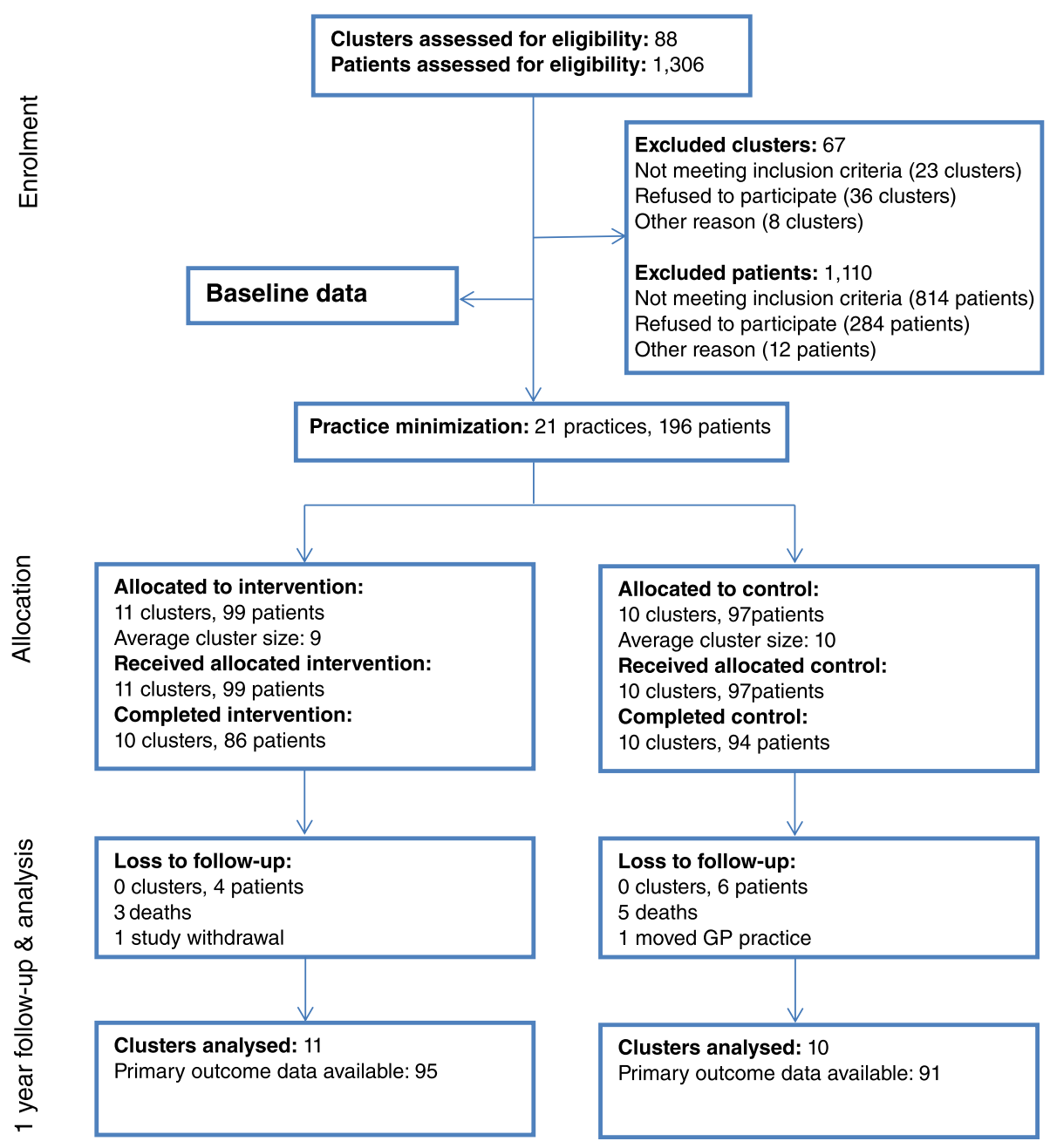

Fig. 1 Flow of practices and patients through study

instances of PIP in the control group $(P=0.38)$. The majority $(81.2 \%)$ had only 1 new PIP. The majority of new prescriptions identified as potentially inappropriate were proton pump inhibitors (44.1\%).

\section{Health service utilisation}

In terms of health service utilisation, there were no statistically significant differences between intervention and control groups. Patients in the control group had an average of $11.7 \mathrm{GP}$ visits compared to 12.2 in the intervention group over the 12-month period. Just over $20 \%$ of intervention and control groups had an in-patient stay.

\section{Discussion}

Using 1-year follow-up, we demonstrated that the significant decreases in PIP rates achieved during our intervention were sustained once it was discontinued.

Table 2 Proportion of patients with PIP at 1-year follow-up

\begin{tabular}{llll}
\hline Characteristic & Intervention N (\%) & Control N (\%) & Adjusted odds ratio $(95 \%$ Cl) \\
\hline PIP at baseline & $99(100)$ & $97(100)$ & \\
PIP at 1-year follow-up & $51(51)$ & $74(76)$ & $0.28(0.11$ to 0.76$)$ \\
No PIP 1-year follow-up & $48(49)$ & $23(24)$ & 0.01 \\
\hline
\end{tabular}

${ }^{2}$ Adjusted for baseline number of PIP, age, gender, number of GPs in practice, practice location 
Our findings substantiate previous findings that have demonstrated that interventions lasting for a limited time period (e.g. educational and multifaceted interventions) can have a long-lasting carry-over effect on improving PIP [17, 18].

An array of factors may have contributed to the sustained effect observed in this study. Firstly, this cohort of older patients experienced few hospitalisations over the 1-year follow-up period, reducing the potential for medication changes or potential errors to arise at these transitions of care (i.e. moving between primary and secondary care) [19]. Hence, prescribing may have been more likely to stay stable over time. Secondly, the effect may also have been maintained due to the medications in question, particularly prescribing of proton pump inhibitors, which may be easier to maintain than other medications. Finally, the intervention itself may be an influential factor. Patients were identified as having PIP which would have been noted in the patient health care record; therefore, at future consultations, GPs may be more cognisant of not restarting the identified PIP. A combination of these factors may have contributed to the persistence of the effect from this one-off intervention.

The OPTI-SCRIPT intervention primarily affected potentially inappropriate proton pump inhibitor prescribing, which was highly prevalent at baseline (60\%). We found no impact on other included medications (e.g. benzodiazepines), likely because of the small numbers of patients exposed to these PIP drugs in this study. The prescription of inappropriate proton pump inhibitor is a substantial driver for the prevalence of PIP both in Ireland [20] and internationally [21-24]. The use of proton pump inhibitors has increased substantially during the past decade internationally, potentially due to increased long-term use for ulcer prophylaxis and perceived lack of serious adverse side effects [24]. However, a significant proportion of this prescribing has been found to be inappropriate, and consequently, there has been an increased focus on reducing inappropriate use to improve patient outcomes and decrease costs $[24,25]$.

A small proportion of the intervention group (13\%) had new PIP at 1-year follow-up, though this was lower than those in the control group (20\%), suggesting some effect on GP prescribing in the intervention practices. The new instances of PIP in intervention practices mainly related to sustained maximal dosage of proton pump inhibitors. From the data presented here, it is unclear if the proton pump inhibitor was indeed appropriate or if it was initiated by the GP or another physician. Proton pump inhibitors initiated in hospitals are frequently continued in primary care, even when inappropriate [26].

Improvements may be observed in control group participants due to reactive effects of being studied (i.e. the possible Hawthorne effect). The control group in this study did alter their prescribing patterns slightly. This may be explained by the fact that during the intervention, they received simple feedback about their patients based on baseline data collection and a report on patient PIP at intervention completion. Feedback has been found to promote slight improvements in professional practice but is most effective when it is provided intensively [27]. In anticipation of this improvements in the control group occurring, we analysed anonymised data from the Primary Care Reimbursement Service (PCRS) pharmacy claim database of dispensed medications (a national prescribing database of GP and pharmacy claims), as a national contemporaneous comparison group. Analysis of this group highlighted that the crude odds of having PIP were lower in the OPTI-SCRIPT intervention group compared to the national comparator group [13].

The study has a number of strengths, including being conducted in 'real-world' practices, the low rate of attrition of from the study (primarily due to the nature of the outcome data) and the completeness of the prescription data. However, there are some limitations including the geographic restriction to a region in Ireland, limiting external validity. In all, $32 \%$ of invited GP practices were recruited which is lower than that reported in other primary care studies [28]. The intervention was effective at decreasing the most prevalent PIP in this study, proton pump inhibitors at maximum therapeutic dosage for more than 8 weeks. Potentially inappropriate proton pump inhibitor is a problem in Ireland and internationally, indicating that this intervention could be generalizable to other settings. However, it has been argued that future studies of PIP should focus on the management of genuinely high-risk medicines (i.e. prescribing likely to lead to adverse clinical outcomes [29]), rather than global lists of potentially inappropriate medications $[30,31]$. It is therefore important to establish the effectiveness of the OPTI-SCRIPT intervention in altering prescribing, other than proton pump inhibitor prescribing.

\section{Conclusions}

Changes in PIP occur against a background of escalating polypharmacy and changes in prescribing patterns of specific medications over time [20]; however, these findings indicate that improvements in prescribing quality can be maintained over time. 


\section{Appendix}

Table 3 Selected prescribing criteria/prescribing indicator [16]

\begin{tabular}{|c|c|c|}
\hline Criteria & Concern & $\begin{array}{l}\text { Estimated } \\
\text { prevalence } \\
\text { Ireland }\end{array}$ \\
\hline PPI for peptic ulcer disease at full therapeutic dosage for $>8$ weeks & $\begin{array}{l}\text { Earlier discontinuation or dose reduction for maintenance/ } \\
\text { prophylactic treatment of peptic ulcer disease, oesophagitis or } \\
\text { GORD indicated }\end{array}$ & $4.1-16.79$ \\
\hline NSAID ( $>3$ months) for relief of mild joint pain in osteoarthritis & $\begin{array}{l}\text { Simple analgesics preferable and usually as effective for pain } \\
\text { relief }\end{array}$ & $1.1-8.8 \%$ \\
\hline $\begin{array}{l}\text { Long term (i.e. >1 month), long-acting benzodiazepines, e.g. chlordi- } \\
\text { azepoxide, flurazepam, nitrazepam, chlorazepate and benzodiaze- } \\
\text { pines with long-acting metabolites, e.g. diazepam }\end{array}$ & Risk of prolonged sedation, confusion, impaired balance, falls & $3.0-9.19$ \\
\hline $\begin{array}{l}\text { Any regular duplicate drug class prescription, e.g. } 2 \text { concurrent } \\
\text { opiates, NSAIDs, SSRIs, loop diuretics, and ACE inhibitors. Excludes } \\
\text { duplicate prescribing of drugs that may be required on a PRN basis, } \\
\text { e.g. inhaled beta } 2 \text { agonists (long and short acting) for asthma or } \\
\text { COPD, and opiates for management of breakthrough pain }\end{array}$ & $\begin{array}{l}\text { Optimisation of monotherapy within a single drug class } \\
\text { should be observed prior to considering a new class of drug }\end{array}$ & $2.2-6.0$ \\
\hline TCAs with an opiate or calcium channel blocker & Risk of severe constipation & $0.4-2.0$ \\
\hline Aspirin at dose $>150 \mathrm{mg} /$ day & Increased bleeding risk, no evidence for increased efficacy & $0.1-1.0$ \\
\hline Theophylline as monotherapy for COPD/asthma & Risk of adverse effects due to narrow therapeutic index & $0.6-1.2$ \\
\hline Use of aspirin and warfarin in combination without histamine $\mathrm{H}_{2}$ & High risk of Gl bleeding & $0.3-1.1$ \\
\hline
\end{tabular}
receptor antagonist (except cimetidine because of interaction with warfarin) or PPI

Doses of short-acting benzodiazepines, doses greater than lorazepam (Ativan $\left.{ }^{\oplus}\right), 3$ mg; oxazepam (Serax $\left.{ }^{\oplus}\right), 60$ mg; alprazolam (Xanax $\left.{ }^{\oplus}\right)$, maximums

2 mg; temazepam (Restoril $\left.{ }^{\oplus}\right), 15$ mg; and triazolam (Halcion $\left.{ }^{\circledast}\right)$, $0.25 \mathrm{mg}$

Prolonged use ( $>1$ week) of first generation antihistamines, i.e. diphenydramine, chlorpheniramine, cyclizine, promethazine

Warfarin and NSAID together

Calcium channel blockers with chronic constipation

NSAID with history of peptic ulcer disease or Gl bleeding, unless with concurrent histamine $\mathrm{H}_{2}$ receptor antagonist, $\mathrm{PPI}$ or misoprostol

Bladder antimuscarinic drugs with dementia

TCAs with constipation

Digoxin at a long-term dose $>125 \mu \mathrm{g} /$ day (with impaired renal function)

Thiazide diuretic with a history of gout

Glibenclamide (with type 2 diabetes mellitus)

Aspirin with a past history of peptic ulcer disease without histamine $\mathrm{H} 2$ receptor antagonist or $\mathrm{PPI}$

Prochlorperazine (Stemetil ${ }^{\oplus}$ ) or metoclopramide with parkinsonism

TCAs with dementia

Risk of sedation and anticholinergic side effects

Risk of Gl bleeding

$0.7-1.7 \%$

May exacerbate constipation

Risk of peptic ulcer relapse

Risk of increased confusion, agitation

May worsen constipation

Increased risk of toxicity

May exacerbate gout

Risk of prolonged hypoglycaemia

Risk of bleeding

Risk of exacerbating parkinsonism

TCAs with glaucoma

TCAs with cardiac conductive abnormalities

Risk of worsening cognitive impairment

Likely to exacerbate glaucoma

$<1.0 \%$

Pro-arrhythmic effects

Long-term corticosteroids (>3 months) as monotherapy for rheumatoid arthritis or osteoarthritis

Risk of major systemic corticosteroid side effects

Bladder antimuscarinic drugs with chronic prostatism

Risk of urinary retention

NSAID with heart failure

TCAs with prostatism or prior history of urinary retention

Systemic corticosteroids instead of inhaled corticosteroids for maintenance therapy in COPD/asthma

Risk of exacerbation of heart failure 
Table 3 Selected prescribing criteria/prescribing indicator [16] (Continued)

\begin{tabular}{|c|c|c|}
\hline Bladder antimuscarinic drugs with chronic glaucoma & Risk of acute exacerbation of glaucoma & $<0.01 \%$ \\
\hline NSAID with SSRI & Increased risk of Gl bleed & N/A \\
\hline Bladder antimuscarinic drugs with chronic constipation & Risk of exacerbation of constipation & N/A \\
\hline $\begin{array}{l}\text { Prednisolone (or equivalent) }>3 \text { months or longer without } \\
\text { bisphosphonate }\end{array}$ & Increased risk of fracture & N/A \\
\hline NSAID with ACE-inhibitor & $\begin{array}{l}\text { Risk of kidney failure, particularly if presence of general } \\
\text { arteriosclerosis, dehydration or concurrent use of diuretics }\end{array}$ & N/A \\
\hline NSAID with diuretic & $\begin{array}{l}\text { May reduce the effect of diuretics and worsen existing heart } \\
\text { failure }\end{array}$ & N/A \\
\hline
\end{tabular}

\section{Abbreviations}

$\mathrm{Cl}$, confidence interval; GP, general practitioner; ICGP, Irish College of General Practitioners; OR, odds ratio; PIP, potentially inappropriate prescribing; $R C T$, randomised controlled trial

\section{Acknowledgements}

David Williams, MD; Ronan McDonnell, PhD; and Daniel Clear, MB, contributed to the design of the intervention. Marie Bradley, PhD, contributed to the study design, intervention design and baseline outcome assessment. Mary-Claire Kennedy, B.Sc. (Pharm), conducted the academic detailing with GPs. Nicola Motterlini (RIP) provided the statistical support at the study inception. We thank the HSE-PCRS for supplying the data for national comparison; in particular, we would also like to thank Frank Moriarty, Caitriona Cahir and Kathleen Bennett. We thank all the GP practices and patients who participated in this study.

\section{Funding}

This study is independent research, funded by the Health Research Board (HRB) PhD Scholars Programme in Health Services Research under Grant No. PHD/2007/16 and the HRB Centre for Primary Care Research under Grant no $\mathrm{HRC} / 2007 / 1$

\section{Authors' contributions}

$\mathrm{BC}$, TF, SMS and $\mathrm{CMH}$ contributed to the conception and design of the study. BC collected the data. JAC conducted the outcome assessment. FB provided the statistical support. All authors contributed to the interpretation of findings and the drafting or revising of the manuscript and have given final approval of the version to be published.

\section{Competing interests}

The authors declare that they have no competing interests.

\section{Ethics approval and consent to participate}

This research was performed in accordance with the Declaration of Helsink and approved by the Research Ethics Committee of the Irish College of General Practitioners (ICGP). Informed consent to participate in the study was obtained from all participants.

\section{Author details}

${ }^{1}$ HRB Centre for Primary Care Research, Department of General Practice, Royal College of Surgeons in Ireland (RCSI), 123 St. Stephens Green, Dublin 2, Republic of Ireland. ${ }^{2}$ School of Pharmacy, Queen's University Belfast (QUB), 97 Lisburn Road, Belfast BT9 7BL, Northern Ireland.

\section{Received: 15 March 2016 Accepted: 23 May 2016}

Published online: 02 June 2016

\section{References}

1. Hanlon JT, Schmader KE, Semla TP. Update of studies on drug-related problems in older adults. J Am Geriatr Soc. 2013;61(8):1365-8.
2. $\quad$ Spinewine A, Schmader K, Barber N, Hughes C, Lapane K, Swine C, et al. Appropriate prescribing in elderly people: how well can it be measured and optimised? Lancet. 2007;370:173-84.

3. Gallagher P, Barry P, O'Mahony D. Inappropriate prescribing in the elderly. J Clin Pharm Ther. 2007:32(2):113-21.

4. Cahir C, Fahey T, Teeling M, Teljeur C, Feely J, Bennett K. Potentially inappropriate prescribing and cost outcomes for older people: a national population study. Br J Clin Pharmacol. 2010;69(5):543-52.

5. Opondo D, Eslami S, Visscher S, Rooij S, Verheij R, Korevaar J, et al. Inappropriateness of medication prescriptions to elderly patients in the primary care setting: a systematic review. PLoS ONE. 2012;7(8):e43617.

6. Hill-Taylor B, Sketris I, Hayden J, Byrne S, O'Sullivan D, Christie R. Application of the STOPP/START criteria: a systematic review of the prevalence of potentially inappropriate prescribing in older adults, and evidence of clinical, humanistic and economic impact. J Clin Pharm Ther. 2013;38(5): 360-72.

7. Jano E, Aparasu RR. Healthcare outcomes associated with Beers' criteria: a systematic review. Ann Pharmacother. 2007:41(3):438-47.

8. Hamilton HJ, Gallagher PF, O'Mahony D. Inappropriate prescribing and adverse drug events in older people. BMC Geriatr. 2009;9:5.

9. Forsetlund L, Eike M, Gjerberg E, Vist G. Effect of interventions to reduce potentially inappropriate use of drugs in nursing homes: a systematic review of randomised controlled trials. BMC Geriatr. 2011;11(1):16.

10. Alldred DP, Raynor DK, Hughes C, Barber N, Chen TF and Spoor P. Interventions to optimise prescribing for older people in care homes. Cochrane Database Syst Rev 2013 Issue 2:Art. No.: CD009095.

11. Kaur S, Mitchell G, Vitetta L, Roberts MS. Interventions that can reduce inappropriate prescribing in the elderly: a systematic review. Drugs Aging. 2009:26(12):1013-28.

12. Yourman L, Concato J, Agostini JV. Use of computer decision support interventions to improve medication prescribing in older adults: a systematic review. Am J Geriatr Pharmacother. 2008;6(2):119-29.

13. Clyne B, Smith SM, Hughes CM, Boland F, Bradley MC, Cooper JA, et al. Effectiveness of a multifaceted intervention for potentially inappropriate prescribing in older patients in primary care: a clusterrandomized controlled trial (OPTI-SCRIPT study). Ann Fam Med. 2015; 13(6):545-53.

14. van der Linden CM, Kerskes MC, Bijl AM, Maas HA, Egberts AC, Jansen PA. Represcription after adverse drug reaction in the elderly: a descriptive study. Arch Intern Med. 2006;166(15):1666-7.

15. Clyne B, Bradley M, Hughes C, Clear D, McDonnell R, Williams D, et al. Addressing potentially inappropriate prescribing in older patients: development and pilot study of an intervention in primary care (the OPTISCRIPT study). BMC Health Serv Res. 2013;13(1):307.

16. Clyne B, Bradley MC, Smith SM, Hughes CM, Motterlini N, Clear D, et al. Effectiveness of medicines review with web-based pharmaceutical treatment algorithms in reducing potentially inappropriate prescribing in older people in primary care: a cluster randomized trial (OPTI-SCRIPT study protocol). Trials. 2013;14(1):72.

17. Lopatto J, Keith SW, Del Canale S, Templin M, Maio V. Evaluating sustained quality improvements: long-term effectiveness of a physician-focused 
intervention to reduce potentially inappropriate medication prescribing in an older population. J Clin Pharm Ther. 2014;39(3):266-71.

18. Rognstad S, Brekke M, Fetveit A, Dalen I, Straand J. Prescription peer academic detailing to reduce inappropriate prescribing for older patients: a cluster randomised controlled trial. Br J Gen Pract. 2013;63(613):e554-62.

19. Redmond P, Grimes T, McDonnell R, Boland F, Hughes C and Fahey T. Interventions for improving medication reconciliation across transitions of care (protocol). Cochrane Database Sys Rev 2013(10):Art. No.: CD010791.

20. Moriarty F, Hardy C, Bennett K, Smith SM, Fahey T. Trends and interaction of polypharmacy and potentially inappropriate prescribing in primary care over 15 years in Ireland: a repeated cross-sectional study. BMJ Open. 2015; 5(9):e008656.

21. Wahab M, Nyfort-Hansen K, Kowalski S. Inappropriate prescribing in hospitalised Australian elderly as determined by the STOPP criteria. Int J Clin Pharm. 2012;34(6):855-62.

22. Kara O, Arik G, Kizilarslanoglu MC, Kilic MK, Varan HD, Sumer F, et al. Potentially inappropriate prescribing according to the STOPP/START criteria for older adults, Aging clinical and experimental research. 2015.

23. Bradley M, Motterlini N, Padmanabhan S, Cahir C, Williams T, Fahey T, et al. Potentially inappropriate prescribing among older people in the United Kingdom. BMC Geriatr. 2014;14(1):72.

24. Hamzat H, Sun H, Ford JC, MacLeod J, Soiza R, Mangoni A. Inappropriate prescribing of proton pump inhibitors in older patients effects of an educational strategy. Drugs Aging. 2012;29(8):681-90

25. Reeve E, Andrews JM, Wiese MD, Hendrix I, Roberts MS, Shakib S. Feasibility of a patient-centered deprescribing process to reduce inappropriate use of proton pump inhibitors. Ann Pharmacother. 2015:49(1):29-38.

26. Wermeling M, Himmel W, Behrens G and Ahrens D. Why do GPs continue inappropriate hospital prescriptions of proton pump inhibitors? A qualitative study. Eur J Gen Pract. 2013;0(0):1-7.

27. Ivers N, Jamtvedt G, Flottorp S, Young J, Odgaard-Jensen J, French S, et al. Audit and feedback: effects on professional practice and healthcare outcomes. Cochrane Database Syst Rev 2012; Issue 6:Art. No.: CD000259.

28. Jones R, Jones R, McCowan C, Montgomery A, Fahey T. The external validity of published randomized controlled trials in primary care. BMC Fam Pract. 2009;10(1):5.

29. Gnjidic D, Le Couteur D, Pearson S-A, McLachlan A, Viney R, Hilmer S, et at. High risk prescribing in older adults: prevalence, clinical and economic implications and potential for intervention at the population level. BMC Public Health. 2013;13(1):115.

30. Barnett K, McCowan C, Evans JMM, Gillespie ND, Davey PG, Fahey T. Prevalence and outcomes of potentially inappropriate medicines use in the elderly: cohort study stratified by residence in nursing home or in the community. BMJ Qual Saf. 2011;20:275-81.

31. Guthrie B, McCowan C, Davey P, Simpson CR, Dreischulte T, Barnett K. High risk prescribing in primary care patients particularly vulnerable to adverse drug events: cross sectional population database analysis in Scottish general practice. BMJ. 2011;342.

\section{Submit your next manuscript to BioMed Central and we will help you at every step:}

- We accept pre-submission inquiries

- Our selector tool helps you to find the most relevant journal

- We provide round the clock customer support

- Convenient online submission

- Thorough peer review

- Inclusion in PubMed and all major indexing services

- Maximum visibility for your research

Submit your manuscript at www.biomedcentral.com/submit 